\title{
A comparison of oncologist versus mental health provider attitudes towards standardized and tailored patient-reported outcomes
}

\author{
Salene M. W. Jones ${ }^{*}$ (D), Aliana Gaffney and Joseph M. Unger
}

\begin{abstract}
Background: Patient-reported outcomes (PROs) can be used to monitor patients during treatment. Healthcare provider preferences for individualized vs. standardized PROs have been understudied.

Methods: This study surveyed oncology and mental health providers to compare attitudes towards individualized and standardized PROs. We have developed a method for individualizing PROs, called precision PROs, and the survey specifically assessed preferences for this method. We compared attitudes and preferences by provider type and by whether respondents were current or never users of PROs.

Results: Oncology providers expressed more positive attitudes for standardized PROs in treatment planning compared to mental health providers $(F(1,440)=5.978, p=0.015)$. The interaction between provider type (oncology vs. mental health) and type of PRO (individualized vs. standardized) was not significant for the attitudes about the clinical utility of PROs $(p=0.709)$. When directly asked about the precision PRO approach, oncologists were less likely to prefer standardized items $(O R=0.478, p=0.001)$ or have no preference $(O R=0.445, p=0.007)$ to the precision PRO approach when compared to mental health providers. Qualitative analyses suggested standardized PROs may be simpler or easier to understand whereas individualized PROs better capture patient variability and the unique aspects of each patient's condition. Some mental health providers expressed reticence about letting patients choose how to tailor PROs. Never users of PROs reported more positive attitudes towards individualized measures than standardized measures whereas current users of PROs did not have a difference in attitudes $(p=0.010)$. User status was mostly unrelated to preferences.
\end{abstract}

Conclusion: Results suggest that healthcare provider preference for individualized PROs may differ by medical specialty. How PROs are tailored may need to differ by discipline. This is particularly important given that previous research showing a preference for individualized PROs over standardized was conducted with psychotherapists. Further research on patient preferences for individualized and standardized PROs is warranted as is research on the clinical utility of individualized PROs such as the precision PRO approach.

Keywords: Questionnaires, Measurement-based care, Symptom monitoring

*Correspondence: smjones3@fredhutch.org Fred Hutchinson Cancer Research Center, 1100 Fairview Ave N, Seattle, WA 98109, USA

\begin{abstract}
Introduction
Patient-reported outcomes (PROs) are questionnairebased measures of symptoms and quality of life that come directly from the patient [1]. PROs are an integral part of measurement-based care (MBC) in several aspects of healthcare, including oncology and mental health [2-4]. $\mathrm{MBC}$ involves periodically measuring patients' signs and
\end{abstract}


symptoms, often with PROs, to determine if treatment is working or needs to be changed [5].

In mental health, there has been a long history of tension between nomothetic or standardized measures in which all patients complete the same items with the same interpretation guidelines and idiographic measures that are tailored and individualized to each patient but are difficult to compare between patients or to norms [6]. One reason for exploring methods of individualizing or tailoring PROs in the idiographic approach is the suggestion that these measures are more sensitive to change [7]. Initial research from counselors in 2017 suggested that healthcare providers might prefer PROs that are individualized or tailored to each patient's values and condition [8].

We have developed a method that tailors PRO items and the meaningful change definition to support the use of individualized PROs, dubbed precision PROs [9]. The first part of the Precision PRO approach asks patients or participants to define a personal minimally important difference (MID) for defining treatment response. This personal MID has been tested in a general medical sample [10]. The second part of the Precision PRO approach asks patients or participants to choose which items or symptoms from a PRO are most meaningful to them personally so the content of the PRO is tailored to the individual patient. In addition to the potential issues of provider preference, we developed this method to address the problem with current MID and responder definitions not incorporating individual patient values and preferences [11]. We have recently completed a test of both Precision PRO approaches in people with cancer and pain. The Precision PRO approach defines the MID and treatment response by what is most valuable or meaningful to the individual patient.

The preference of healthcare providers for or against such individualized approaches has not been studied extensively and differences by medical specialty have not been explored. Most studies examine preference or attitudes for standardized, nomothetic methods [12]. To address this gap, we surveyed oncology and mental health providers to determine if preferences for individualized PROs differ by specialty. We also conducted exploratory analyses comparing current users of PROs to providers who never used PROs to determine if individualizing PROs might improve uptake among never users.

\section{Methods}

\section{Participants and procedures}

Participants were recruited through survey panels maintained by Qualtrics in August 2019. Participants' status as either an oncology provider (OP) or mental health provider (MHP) was verified through their National Provider
Identification number. Potential participants were sent an invitation to complete the survey online. When participants came to the survey link, they first read the consent form and then, if they agreed to complete the study, clicked through to the survey. Participants received standard incentives for completing the survey such as store gift cards and airline miles. Typical response rates for Qualtrics panels are 5-12\%. All procedures were reviewed and approved by the Fred Hutchinson Cancer Research Center review board (\#8703).

\section{Measures \\ Attitudes towards standardized and individualized assessment}

To assess provider attitudes towards standardized and individualized PROs, participants completed two subscales from the Attitudes towards Standardized Assessment and Attitudes towards Individualized Assessment scales [8], two validated and reliable measures of provider attitudes. Each of these attitudes scales has a clinical utility subscale (8 items, 6 reverse scored) and a treatment planning subscale (5 items). Each item is rated on a fivepoint scale from strongly disagree to strongly agree. Subscale scores are created by averaging the items and we specifically only scored the measures if participants had responded to at least half the items for the subscale. The two scales have the same 13 items and two subscales except one references standardized measures and the other references individualized measures. For example, an item from the treatment planning subscale is "Standardized progress measures help identify when treatment is not going well" on the standardized version and "Individualized progress measures help identify when treatment is not going well" on the individualized version. Items are averaged with higher scores indicating more positive views of either standardized or individualized measures.

\section{Preference for precision PRO approach}

To assess participants preferences for the precision PRO approach specifically, two close-ended and two openended questions were asked (see Additional file 1). The standard PRO approach was based on traditional nomothetic approaches $[6,13]$. The first close-ended question described the standard minimally important difference (MID) approach and precision PRO MID then asked participants which approach they preferred. The standard version was scored on a scale of 0 to 100 , where an increase or decrease of five points was considered meaningful for all patients. The Precision PRO version was also scored on a scale of 0 to 100 but patients define for themselves what increase or decrease in symptoms is meaningful for the patient personally, sometimes in 
consultation with a physician. The second close-ended question described a standard symptom PRO and the precision PRO approach for tailoring items with participants again asked to indicate which they preferred. The standard version had 5 fixed items (symptoms) that were answered by all patients. In the precision PRO version, however, participants chose 5 items (symptoms) out of a list of 30 possible items or symptoms that were most applicable to them personally. For both close-ended questions, 'don't know', 'neither' and 'both' options were provided (see Additional File 1). The two open ended questions asked participants for their reasons for the close-ended question responses.

\section{Characteristics}

The first question on the survey asked participants to indicate if they had never used PROs in treatment monitoring, currently use PROs, or used to use PROs but no longer do. Because few participants $(n=21)$ reported formerly using PROs, these participants were excluded from analyses on user type. However, former users were included in analyses examining provider type (oncology vs. mental health). Participants also reported various demographic and professional characteristics.

\section{Quantitative analysis}

For the Attitudes toward Standardized/Individualized Assessment subscales, we conducted a series of analyses of covariance (ANCOVA). Provider type was a between-subjects factor and type of PRO (standardized, individualized) was a within-subjects factor in the first set of ANCOVAs. In the second set of ANCOVAs, user type (never vs. current) replaced provider type as the between-subjects factor. Age and gender were included as covariates. For the preferences questions, responses were coded into three categories: precision PRO preference (reference); standard PRO preference; and no preference (prefer both, don't know, neither, skipped question). The three preference categories were used as the outcome variable in a multinomial regression. The predictor of interest for the first set of multinomial regressions was provider type while covarying for age and gender. The predictor of interest for the second set of multinomial regressions was user type (never vs. current) while covarying for age and gender.

\section{Qualitative analysis}

After the preference questions, participants provided an explanation supporting their choice. The text for the explanations were coded by two members of the research team using a content analysis approach. The codebook was developed by coder 1 after reviewing the responses given by OPs and MHPs. Next, coder 2 coded each of the 450 responses for the individualized-items question into one or more of the 17 categories and coded each response for the individualized-MID question into one or more of the 15 categories. Coder 1 double coded $10 \%$ of responses for each question to ensure reliability. The two coders discussed major differences and agreed upon revisions to both the codebook and responses. Codes did not have to be mutually exclusive.

\section{Results}

The demographics of the sample $(n=450)$ were consistent with being drawn from a healthcare provider population (Table 1). The average age was 51.1 years old and most participants were White $(\mathrm{n}=313,69.6 \%)$ or Asian $(n=107,23.8 \%)$. Slightly more than two-thirds of the sample was male $(n=308,68.4 \%)$. Most respondents currently used PROs to monitor treatment $(\mathrm{n}=347,77.1 \%)$.

\section{Quantitative analyses}

\section{Attitudes Towards Assessment Scale}

Overall, healthcare providers reported neutral attitudes towards assessment scales. Means ranged from 2.95 to 3.61 (Fig. 1) with 3 on the scale representing neither positive nor negative attitude towards assessment scales. Oncology and mental health providers did not differ in their attitudes towards assessment scales for clinical utility $(F(1,442)=0.139, p=0.709$; Fig. 1a). However, there

Table 1 Sample description

\begin{tabular}{lc}
\hline Characteristic & Mean (SD) or N (\%) \\
\hline Age, years & $51.1(11.4)$ \\
Gender & \\
Male & $308(68.4 \%)$ \\
Female & $129(28.7 \%)$ \\
Other, declined to answer & $13(2.9 \%)$ \\
Race/Ethnicity & \\
White & $313(69.6 \%)$ \\
Black or African-American & $8(1.8 \%)$ \\
Hispanic & $16(3.6 \%)$ \\
Asian & $107(23.8 \%)$ \\
Native American, Pacific Islander, Other & $15(3.3 \%)$ \\
Provider type & \\
Mental Health & $250(55.6 \%)$ \\
Oncology & $200(44.4 \%)$ \\
Has a doctorate-level medical degree & $418(92.9 \%)$ \\
Has non-medical doctorate degree & $47(10.4 \%)$ \\
PRO use & $82(18.2 \%)$ \\
Never user & $21(4.7 \%)$ \\
Former user & $347(77.1 \%)$ \\
Current user & $81.3(76.6)$ \\
Number of patients seen per week &
\end{tabular}




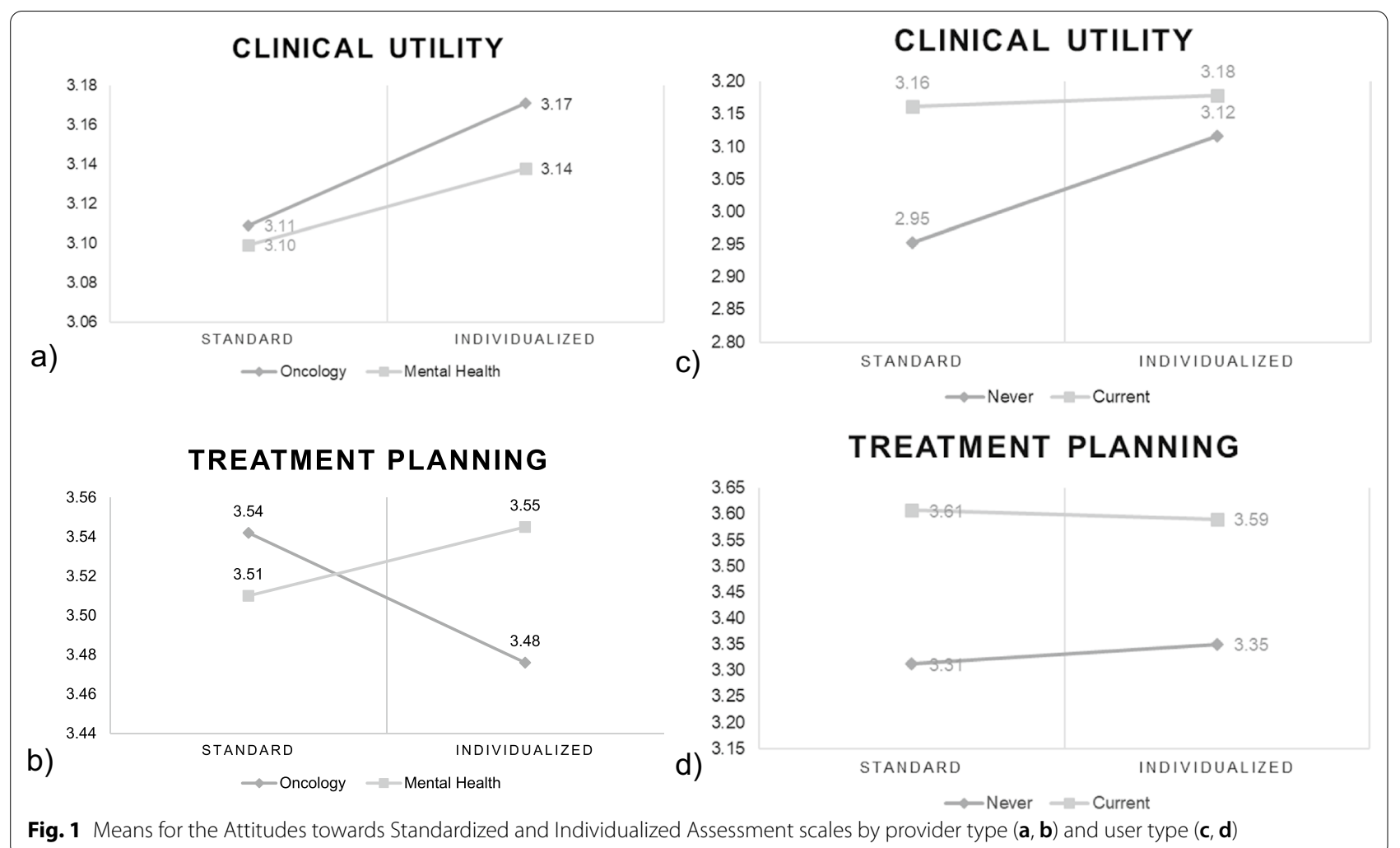

was a significant interaction between provider type and standardized vs. individualized assessment in treatment planning $(F(1,440)=5.978, p=0.015$; Fig. 1b). Oncology providers tended to have more positive attitudes towards standardized assessments whereas mental health providers did not have a more positive attitude towards standardized assessments. For user type, there was a significant interaction between user type and standardized vs. individualized assessment in clinical utility $(\mathrm{F}(1,421)=6.720$, $\mathrm{p}=0.010$; Fig. 1c) such that never users tended to report more positive attitudes towards individualized measures whereas current users reported no difference in attitudes. For user type and treatment planning, there was a main effect in which current users reported more positive attitudes than never users $(\mathrm{F}(1,419)=11.833, \mathrm{p}=0.001$; Fig. 1d). No other main effects or interactions were significant ( $p$ 's $>0.05)$.

\section{Direct comparison of precision PRO and standard approaches}

When asked directly about the two different aspects of the Precision PRO approach, between 21 to $37 \%$ of each subgroup (oncology provider, mental health provider, never user, current user) expressed a preference for the Precision PRO approach (Fig. 2). Provider type (oncology vs. mental health) was not related to preference for the
Precision PRO method of tailoring the MID (p's >0.05). Being an oncology provider versus a mental health provider was associated with lower odds of expressing no preference (odds ratio $(\mathrm{OR})=0.445, \mathrm{p}=0.007$ ) or a preference for standardized, nomothetic PROs $(\mathrm{OR}=0.478$, $\mathrm{p}=0.001$ ) compared to the individualized Precision PRO approach of tailoring items. Never users were more likely to express no preference compared to current users $(\mathrm{OR}=2.351, \mathrm{p}=0.009)$ for the Precision PRO method of tailoring the MID, but otherwise user status was unrelated to preferences for Precision PROs vs. standard, nomothetic PROs (p's >0.05).

\section{Qualitative analysis Individualized items}

Results from qualitative analyses for the individualized items of the Precision PRO approach are reported in Table 2. Of the 450 respondents, 46 (10.2\%) skipped the text question and $20(4.4 \%)$ provided answers that were not codable. Most participants described a preference of PRO version within three main categories: one version is easier or simpler $(n=124)$, one version is too complicated $(\mathrm{n}=42)$, and the Precision PRO approach to items captures patient variability $(n=69)$. Some participants cited that they had no preference of PRO version or that they needed more information $(n=34)$ to decide what 


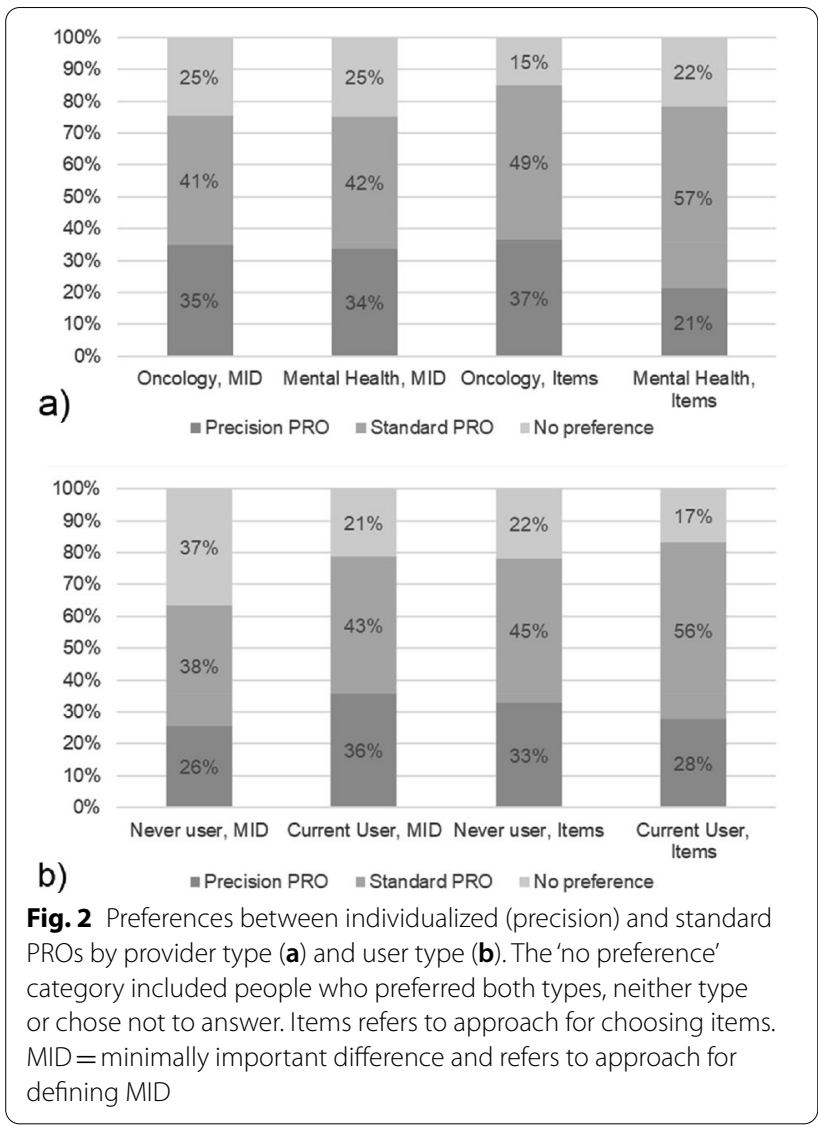

was best for their practice. Overall, participants who preferred the standard PRO approach to items $(n=240)$ said it was simpler and easier. Those who chose the Precision PRO approach to items $(n=126)$, favored it because it was more individualized and had more patient variability. Other participants found PROs ineffective in general and some MHPs thought patients shouldn't chose items.

\section{Individualized MID}

Results from qualitative analyses for individualized, Precision PRO MID approach are reported in Table 3. Of the 450 respondents $50(11.1 \%)$ skipped the text questions and $44(9.8 \%)$ provided answers that were not codable. Most participants described a preference of PRO version within four main categories: one version is easier or simpler $(\mathrm{n}=82)$, the Precision PRO captures patient variability $(n=59)$, one version is perceived as less biased $(n=45)$, and one version uses patient input $(n=41)$. Most participants preferred the standard PRO $(n=185)$ because it was less complicated. However, those who chose the Precision PRO $(n=154)$, explained that it had more variability and was more individualized for the patient. Some participants did not think patients would know what is meaningful $(n=16)$. Other participants either did not have a preference between the two PRO versions, or they found PROs ineffective in general.

\section{Confusion}

Qualitative analysis revealed that some participants found the four versions of PROs to be confusing. The most frequent reasons for confusion were that some participants did not understand the difference of the PRO versions, while others made contradictory remarks to their PRO version selection. Those who stated that they were confused stated they would need more information about the specifics for each version of PRO.

\section{Discussion}

This study examined preferences for individualized PRO measures, such as our Precision PRO method, comparing oncology providers to mental health providers, and comparing never users to current users of PROs. Quantitative results suggested that oncology providers had a more positive general attitude towards standardized measures over individualized measures when compared to mental health providers but oncology providers preferred part of the individualized precision PRO approach, specifically tailoring items, compared to mental health providers. This discrepancy in results for oncology providers could be due to the measures used. The attitude questions were fairly general whereas the preference questions provided specific methods for tailoring PROs within the Precision PRO approach. Some professions like oncology may generally prefer standardized measures but be open to tailoring on specific aspects of PROs, such as which items to administer. Never users may also prefer individualized measures to standardized measures. The Precision PRO approach was preferred by one fifth to one third of the sample, suggesting this approach should be investigated further for patient preference and clinical utility. Qualitative results suggested provider preference was due to either a desire for simplicity or to capture the unique aspects of each patient.

Previous research has shown a preference for individualized measures among mental health providers [8]. Our results, comparing mental health and oncology providers, suggest that how measures are individualized may need to differ by discipline. For oncology, having patients tailor the PRO items may be most important whereas for mental health, having the clinician tailor the items or using standardized items may be most important. However, the overall assessment of individualized measures was neither positive nor negative and, given that standardized measures are what is typically used in clinical care [13], healthcare providers may become more open to 


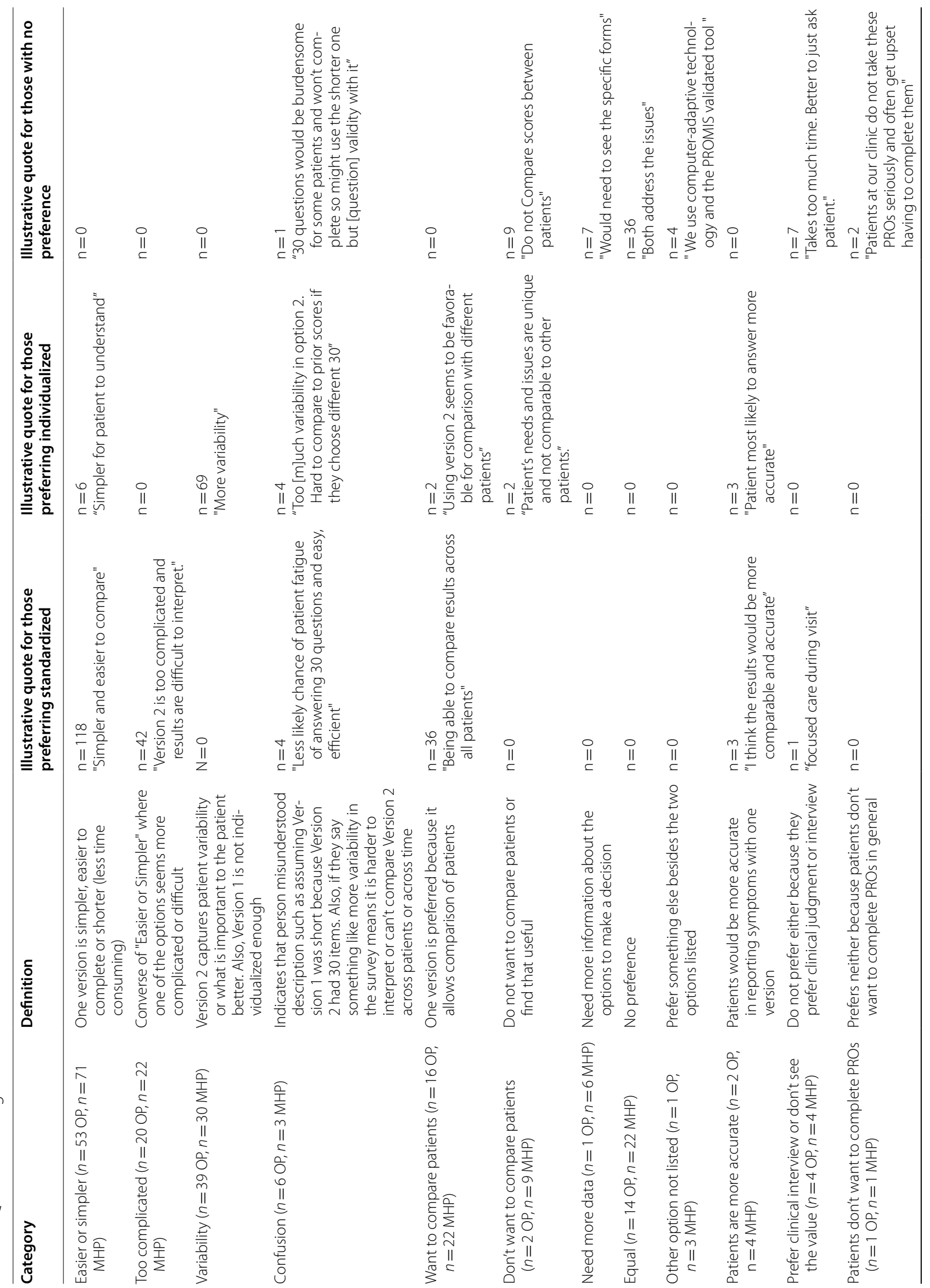




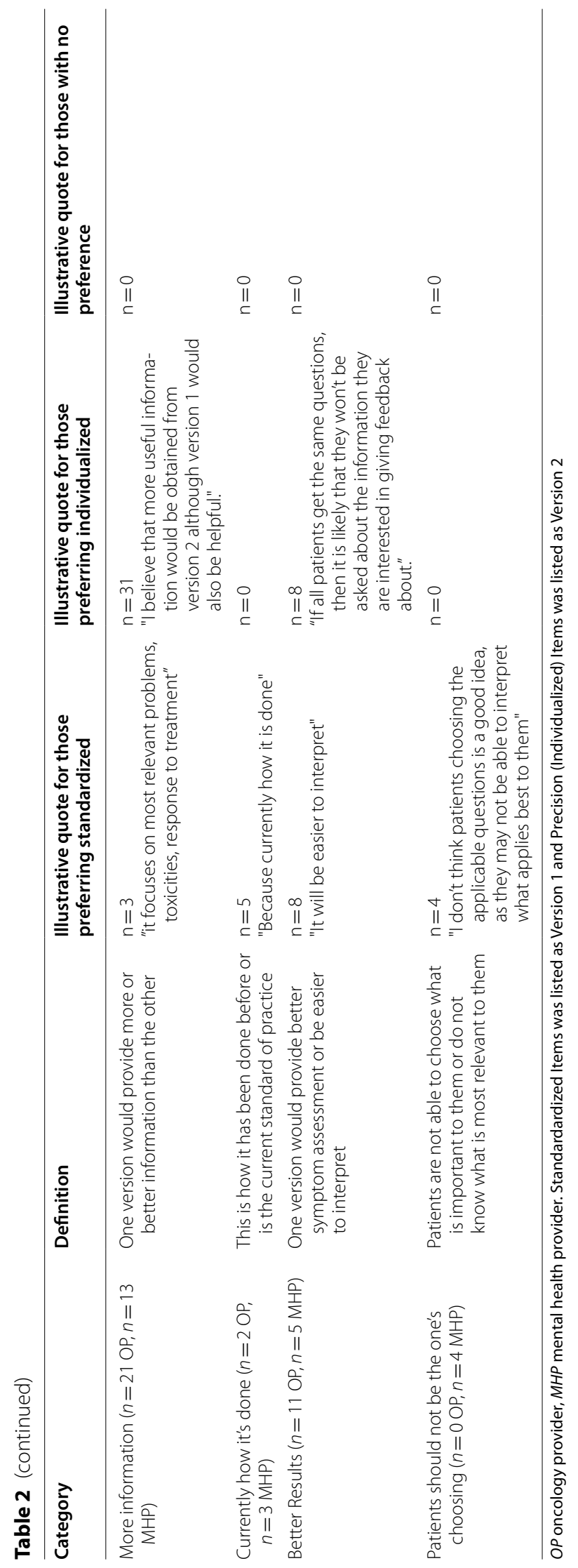




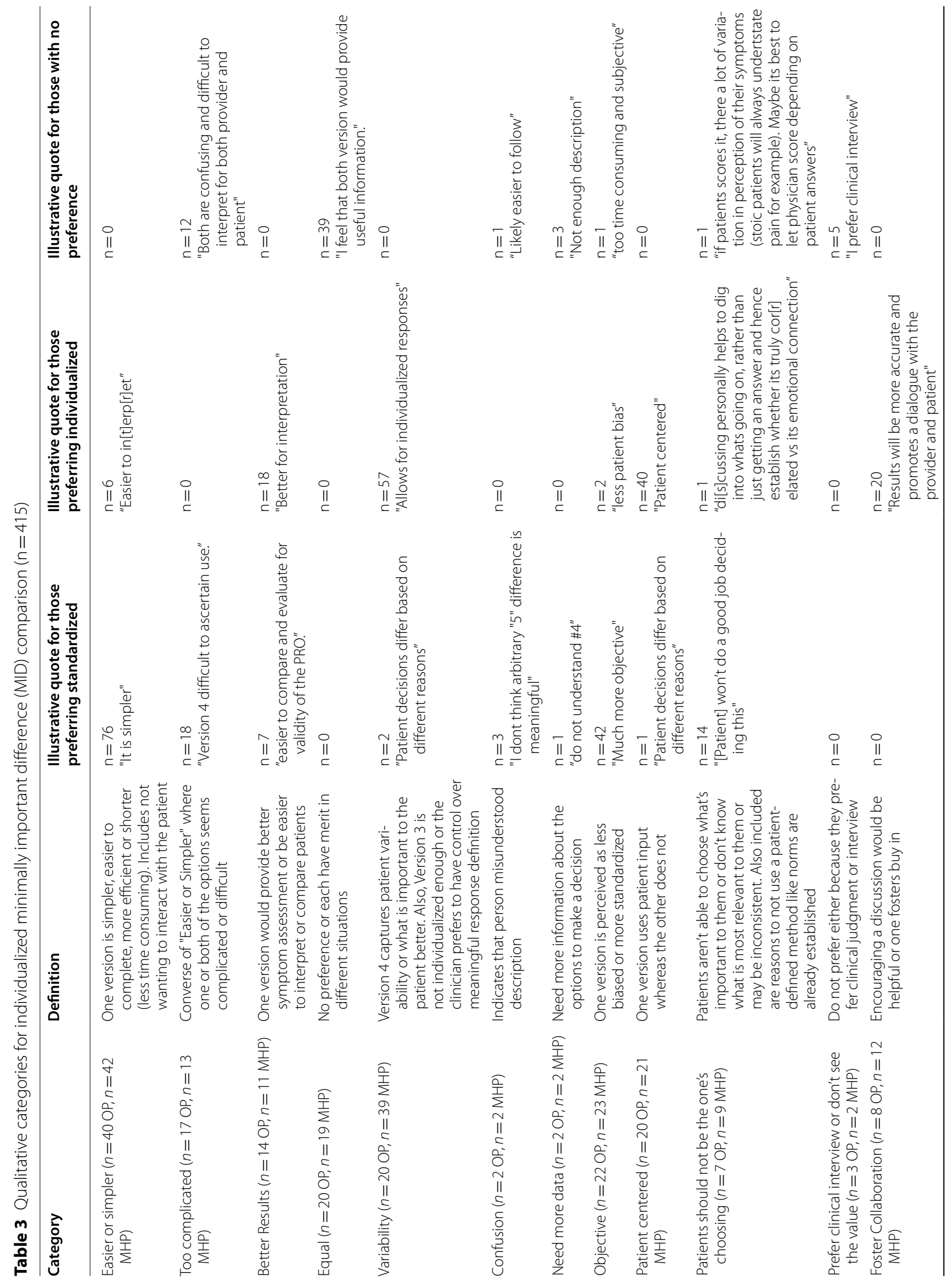


individualized measures as they learn more and research progresses.

The limitations of this study warrant comment. This was a convenience sample of mostly physicians. There may have been a responder bias for current PRO users to respond given the high rate of current use in the sample. A subset of respondents also reported confusion about some of the questions and the discrepant results for oncology suggests healthcare providers may have had some difficulty understanding the idea of tailoring PROs.

\section{Conclusions}

Despite the limitations, our results suggest directions for further research on individualized PROs. First, this study only examined provider preference, showing some preference for individualized PROs, but ultimately patient preference is paramount and needs to be examined in future studies. This study showed discipline might moderate provider preference for individualized PROs but did not examine the utility of these measures. Additional research is needed to determine how individualized PROs, such as Precision PROs, compare to standard PROs in predicting outcomes such as mortality and whether individualized PROs can improve clinical care and research.

\section{Supplementary Information}

The online version contains supplementary material available at https://doi. org/10.1186/s41687-021-00352-8.

Additional file 1. Supplemental Material

\section{Acknowledgements}

The authors would like to thank the participants.

\section{Authors' contributions}

SMWJ—study conceptualization, data collection, data analysis, drafting manuscript. AG—data analysis, manuscript revisions. JMU—-study conceptualization, manuscript revisions. All authors read and approved the final manuscript.

\section{Funding}

Institutional funding was used for this study.

\section{Availability of data and materials}

De-identified data is available upon reasonable request.

\section{Declarations}

Ethics approval and consent to participate

All study activities were reviewed by the applicable institutional review boards and all participants provided informed consent.
Consent for publication

Not applicable.

\section{Competing interests}

The authors declare that they have no competing interests.

Received: 10 May 2021 Accepted: 16 Auqust 2021

Published online: 24 August 2021

\section{References}

1. Administration FaD (2009) Guidance for industry patient-reported outcome measures: use in medical product development to support labeling claims. In: Services HaH (ed) Food and Drug Administration, Rockville

2. Basch E, Deal AM, Dueck AC, Scher HI, Kris MG, Hudis C et al (2017) Overall survival results of a trial assessing patient-reported outcomes for symptom monitoring during routine cancer treatment. JAMA 318(2):197-198

3. Basch E, Deal AM, Kris MG, Scher HI, Hudis CA, Sabbatini P et al (2016) Symptom monitoring with patient-reported outcomes during routine cancer treatment: a randomized controlled trial. J Clin Oncol 34(6):557-565

4. Fortney J, Sladek R, Unützer J, Kennedy P, Harbin H, Emmet B, et al (2015) Fixing behavioral health care in America: a national call for measurementbased care in the delivery of behavioral health services. The Kennedy Forum

5. Scott K, Lewis CC (2015) Using measurement-based care to enhance any treatment. Cogn Behav Pract 22(1):49-59

6. Lyon AR, Connors E, Jensen-Doss A, Landes SJ, Lewis CC, McLeod BD et al (2017) Intentional research design in implementation science: implications for the use of nomothetic and idiographic assessment. Transl Behav Med 7(3):567-580

7. Ashworth M, Guerra D, Kordowicz M (2019) Individualised or standardised outcome measures: a co-habitation? Admin Policy Ment Health Ment Health Serv Res 46(4):425-428

8. Jensen-Doss A, Smith AM, Becker-Haimes EM, Mora Ringle V, Walsh LM, Nanda M et al (2018) Individualized progress measures are more acceptable to clinicians than standardized measures: results of a national survey. Adm Policy Ment Health 45(3):392-403

9. Jones SMW, Ramsey SD, Unger JM (2020) An editorial on tailoring patientreported outcomes to the individual patient: Precision PROs (preprint)

10. Jones SMW, Du Y, Bell-Brown A, Bolt K, Unger JM (2020) Feasibility and validity of asking patients to define individual levels of meaningful change on patient-reported outcomes. J Patient Cent Res Rev 7(3):239-248

11. Hays RD, Peipert JD (2021) Between-group minimally important change versus individual treatment responders. Qual Life Res

12. Jensen-Doss A, Haimes EM, Smith AM, Lyon AR, Lewis CC, Stanick CF et al (2018) Monitoring treatment progress and providing feedback is viewed favorably but rarely used in practice. Adm Policy Ment Health Ment Health Serv Res 45(1):48-61

13. Jacobsen PB, Ransom S (2007) Implementation of NCCN distress management guidelines by member institutions. J Natl Compr Canc Netw 5(1):99-103

\section{Publisher's Note}

Springer Nature remains neutral with regard to jurisdictional claims in published maps and institutional affiliations. 